Research Article

\title{
Stable Copper Tin Sulfide Nanoflower Modified Carbon Quantum Dots for Improved Supercapacitors
}

\author{
Zhen Bi, ${ }^{1}$ Lanyan Huang, ${ }^{1}$ Chaoqun Shang $\mathbb{D}^{1}{ }^{1}$ Xin Wang $\mathbb{D},{ }^{1,2}$ and Guofu Zhou $\mathbb{D}^{1,2}$ \\ ${ }^{1}$ National Center for International Research on Green Optoelectronics, South China Normal University, \\ Guangzhou 510006, China \\ ${ }^{2}$ International Academy of Optoelectronics at Zhaoqing, South China Normal University, Zhaoqing 526060, China
}

Correspondence should be addressed to Chaoqun Shang; chaoqun.shang@ecs-scnu.org and Xin Wang; wangxin@scnu.edu.cn

Received 14 March 2019; Accepted 22 May 2019; Published 4 June 2019

Academic Editor: Cláudia G. Silva

Copyright (C) 2019 Zhen Bi et al. This is an open access article distributed under the Creative Commons Attribution License, which permits unrestricted use, distribution, and reproduction in any medium, provided the original work is properly cited.

\begin{abstract}
Copper tin sulfides (CTSs) have widely been investigated as electrode materials for supercapacitors owing to their high theoretical pseudocapacitances. However, the poor intrinsic conductivity and volume change during redox reactions hindered their electrochemical performances and broad applications. In this study, carbon quantum dots (CQDs) were employed to modify CTSs. The structures and morphologies of obtained materials were characterized by X-ray diffraction (XRD) and transmission electron microscopy (TEM). XRD revealed CTSs were composed of $\mathrm{Cu}_{2} \mathrm{SnS}_{3}$ and $\mathrm{Cu}_{4} \mathrm{SnS}_{4}$, and TEM suggested the decoration of CQDs on the surface of CTSs. With the decoration of CQDs, CTSs@CQDs showed a remarkable specific capacitance of $856 \mathrm{~F} \cdot \mathrm{g}^{-1}$ at $2 \mathrm{mV} \cdot \mathrm{s}^{-1}$ and a high rate capability of $474 \mathrm{~F} \cdot \mathrm{g}^{-1}$ at $50 \mathrm{mV} \cdot \mathrm{s}^{-1}$, which were superior to those of CTSs ( $851 \mathrm{~F} \cdot \mathrm{g}^{-1}$ at $2 \mathrm{mV} \cdot \mathrm{s}^{-1}$ and $192 \mathrm{~F} \cdot \mathrm{g}^{-1}$ at $50 \mathrm{mV} \cdot \mathrm{s}^{-1}$, respectively). This was mainly ascribed to incorporation of carbon quantum dots, which improved the electrical conductivity and alleviated volume change of CTSs during charge/ discharge processes.
\end{abstract}

\section{Introduction}

Supercapacitors with high power density, superior efficiency, and long cycle life have attracted increasing attention in energy storage devices $[1,2]$. Compared to conventional carbon materials based on electrical double-layer capacitance, metal oxides/sulfides and conductive polymers possess preeminent specific capacitance due to faradaic pseudocapacitance $[3,4]$.

Among potential electrode materials for pseudocapacitors, transition metal sulfides, including $\mathrm{CuS}$ [5-9], $\mathrm{Cu}_{2} \mathrm{~S}$ [10], $\mathrm{SnS}_{2}[3,11-13]$ and $\mathrm{SnS}$ [14] have extensively been investigated owing to their earth abundance, low cost, environmental friendliness, and high theoretical capacitance. On the contrary, CTSs have particularly been applied in conversion and storage devices [15-18]. However, CTSs as electrode materials for supercapacitors still suffer from poor electrical conductivity and large volume change during redox reactions, leading to moderate electrochemical performances. Herein, CQDs were employed to decorate CTSs and yield CTSs@CQDs with enhanced electrochemical performances in terms of rate capability and cycling stability.

\section{Experimental}

2.1. Chemicals. Tin chloride pentahydrate $\left(\mathrm{SnCl}_{4} \cdot 5 \mathrm{H}_{2} \mathrm{O}\right)$, copric chloride dihydrate $\left(\mathrm{CuCl}_{2} \cdot 2 \mathrm{H}_{2} \mathrm{O}\right)$, and polyethylene glycol (average Mn 200) were purchased from Aladdin (Shanghai, China). Thioacetamide were purchased from Sinopharm Chemical Regent Co., Ltd. (Shanghai, China). All chemicals were of analytical grade.

2.2. Synthesis of CTS Microspheres. All reactants and solvents were used directly as received without further purification. In the typical procedure, $1 \mathrm{mmol} \mathrm{SnCl}_{4} \cdot 5 \mathrm{H}_{2} \mathrm{O}$, $2 \mathrm{mmol} \mathrm{CuCl}_{2} \cdot 2 \mathrm{H}_{2} \mathrm{O}$, and $3 \mathrm{mmol}$ thioacetamide were successively added into a $50 \mathrm{~mL}$ beaker containing $30 \mathrm{~mL}$ PEG-200. The mixture was then heated at $60^{\circ} \mathrm{C}$ for $40 \mathrm{~min}$ 
under constant magnetic stirring to form a black solution. The obtained mixture was transferred to a $50 \mathrm{~mL}$ Teflonlined stainless steel autoclave and heated at $180^{\circ} \mathrm{C}$ for $16 \mathrm{~h}$. After cooling to room temperature, the mixture was centrifuged, washed three times with deionized water and ethanol, and dried in a vacuum oven at $60^{\circ} \mathrm{C}$ for $12 \mathrm{~h}$ to yield the precursor. To obtain CTS microspheres, the precursor was annealed at $400^{\circ} \mathrm{C}$ for $4 \mathrm{~h}$ under Ar atmosphere.

\subsection{Synthesis of CTSs@CQDs Nanoparticles.CTSs@CQDs} nanoparticles were prepared by adding $20 \mathrm{mg}$ of assynthesized CTSs microspheres and $0.2 \mathrm{~g}$ carbon quantum dots solution into $1.8 \mathrm{~g}$ acetone under constant stirring at room temperature for $1 \mathrm{~h}$. The CTSs@CQDs were obtained after drying at $80^{\circ} \mathrm{C}$ to evaporate remaining acetone.

2.4. Characterization. The crystallinity and composition of the materials were identified by XRD. The morphologies were viewed by TEM, high-resolution TEM (HRTEM), and energy dispersive X-ray (EDX).

2.5. Electrochemical Measurements. The electrochemical performances of CTSs@CQDs were measured in a threeelectrode system connected to a CHI660E electrochemical workstation. Pt was used as counter, $\mathrm{Hg} / \mathrm{HgO}$ as reference, and CTSs@CQDs as the working electrode. The working electrodes were prepared by mixing CTSs@CQDs, carbon black, and PTFE at a weight ratio of $85: 5: 10$. The mixture was ground in a mortar to form slurry, which was then thin sliced with a glass rod. The working electrodes were harvested by pressing the thin slice ( 1 mg) on the nickel foam current collector.

\section{Results and Discussion}

3.1. Structure and Morphology. Figure 1 shows the XRD patterns of CTSs and CTSs@CQDs. Obviously, CTSs are composited of predominant $\mathrm{Cu}_{2} \mathrm{SnS}_{3}$ (JCPDS \# 89-2877) and partial $\mathrm{Cu}_{4} \mathrm{SnS}_{4}$ (JCPDS \# 27-0196), where the sharp diffraction peaks at $2 \theta=28.5^{\circ}, 32.8^{\circ}, 47.5^{\circ}, 56.4^{\circ}, 68.6^{\circ}$, and $76.3^{\circ}$ were assigned to (111), (200), (220), (311), (400), and (331) of $\mathrm{Cu}_{2} \mathrm{SnS}_{3}$, respectively [19]. The composition of CTSs provided abundant valence states, which was beneficial to the redox reactions during charging/discharging processes. With the modification of CQDs, CTSs@CQDs displayed a similar XRD pattern to that of CTSs, demonstrating the CQDs were attached on the surface of CTSs without changing the crystalline nature of CTSs [20,21].

TEM analysis was employed to investigate the micro/ nanostructures of CTSs and CTSs@CQDs. As displayed in Figures 2(a) and 2(c), CTSs with an interesting flower structure were assembled by nanosheets. The typical HRTEM image of CTSs (Figure 2(e)) specified the lattice fringe spacing was estimated to be around $0.31 \mathrm{~nm}$, in good accordance with the (111) plane for cubic CTSs [18]. With the modification of CQDs, CTSs@CQDs exhibited the nanoflower-like structure with a slight change (Figures 2(b) and 2(d)), further demonstrating the decoration of CQDs.

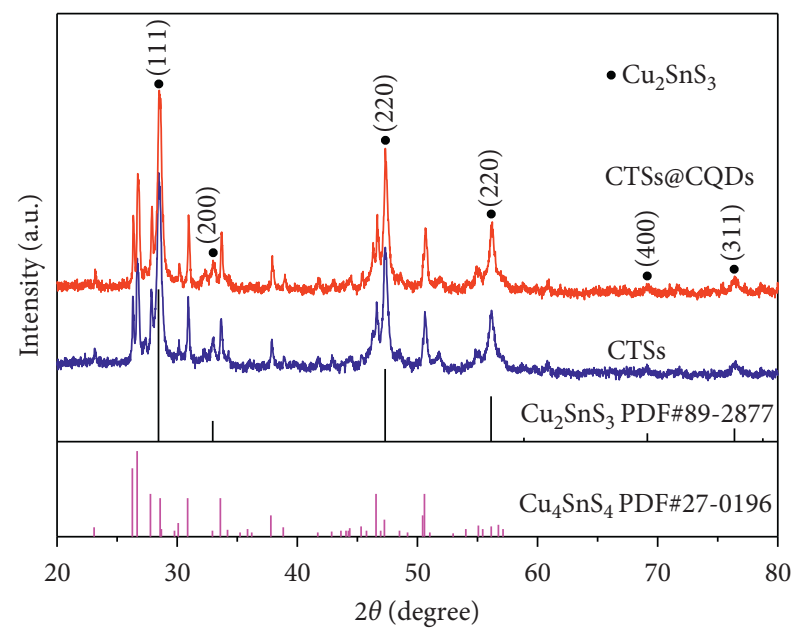

FIgure 1: XRD patterns of CTSs and CTSs@CQDs.

As illustrated in Figure 2(f), the nanosheet was covered by CQDs (red rings), which was beneficial to fast electron transport and alleviate volume variation. In the TEM and corresponding EDX mapping results (Figure $2(\mathrm{~g})$ ), $\mathrm{Cu}, \mathrm{Sn}$, and $\mathrm{S}$ were homogeneously distributed. Besides, the CQDs were uniformly distributed throughout the nanoflower-like structures, which could considerably improve the electrochemical performance of supercapacitors [22, 23].

3.2. Electrochemical Performance. As shown in Figure 3(a), the CV curves of CTSs at a scan rate of $50 \mathrm{mV} \cdot \mathrm{s}^{-1}$ exhibited two pairs of significant redox peaks, demonstrating obvious pseudocapacitive behaviour. The redox peaks at $0 \mathrm{~V} /-0.85 \mathrm{~V}$ may correspond to the redox reaction between $\mathrm{Cu}_{2} \mathrm{SnS}_{3}$ and $\mathrm{Cu}_{2} \mathrm{SnS}_{3} \mathrm{OH}$. Another pair of redox peaks at $-0.31 \mathrm{~V} /-0.43 \mathrm{~V}$ might be attributed to the redox reaction of $\mathrm{Cu} / \mathrm{Cu}^{+}[5,6]$. As a fair comparison, the CV curves of CTSs@CQDs at various scan rates were depicted in Figure 3(b). The specific capacitances of the electrodes can be calculated using the following equation [24-27]:

$$
C_{\mathrm{m}}=\frac{\int i d V}{2 m v \Delta V},
$$

where $C_{\mathrm{m}}$ is the specific capacitance, $i$ is the charge/discharge current, $m$ is the mass of the active electrode material, $v$ is the scan rate, and $\Delta V$ is the voltage window.

Here, the calculated specific capacitances of CTSs and CTSs@CQDs were summarized in Figure 3(c). Interestingly, at a low scan rate of 2 and $5 \mathrm{mV} \cdot \mathrm{s}^{-1}$, CTSs and CTSs@CQDs showed similar specific capacitances, which could be attributed to the nanoflower nanostructure, ensuring enough electrolyte penetration and fast ion transport. However, with the increasing scan rate, CTSs@CQDs demonstrated enhanced electrochemical performance than that of CTSs owing to the modification of CQDs. Even at a high scan rate of $50 \mathrm{mV} \cdot \mathrm{s}^{-1}$, CTSs@CQDs delivered a specific capacitance of $474 \mathrm{~F} \cdot \mathrm{g}^{-1}$, which was over two times to that of CTSs $\left(192 \mathrm{~F}^{-1} \mathrm{~g}^{-1}\right.$ ), further confirming the important role of CQDs. The cycling stability of CTSs and CTSs@CQDs is depicted in 


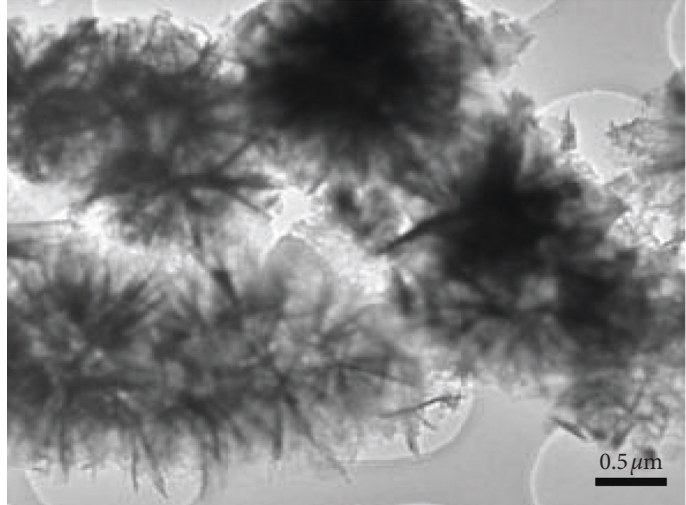

(a)

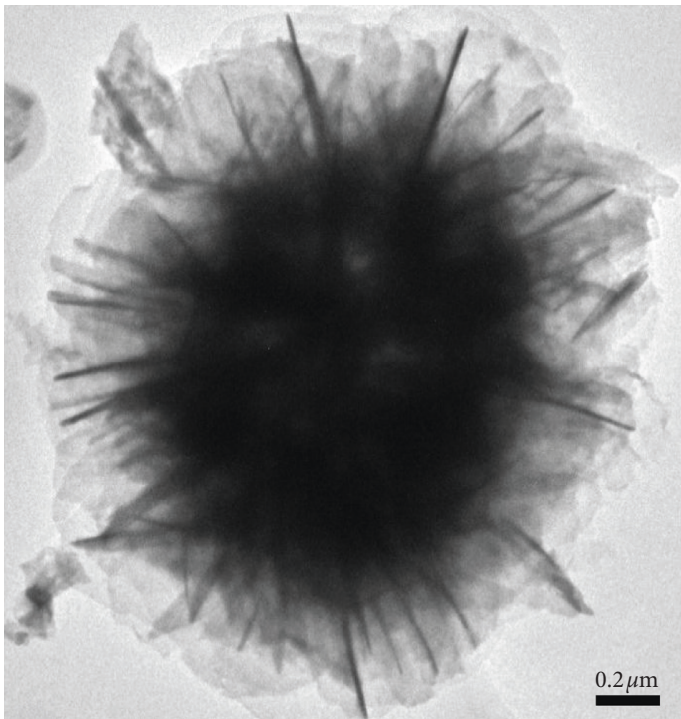

(c)

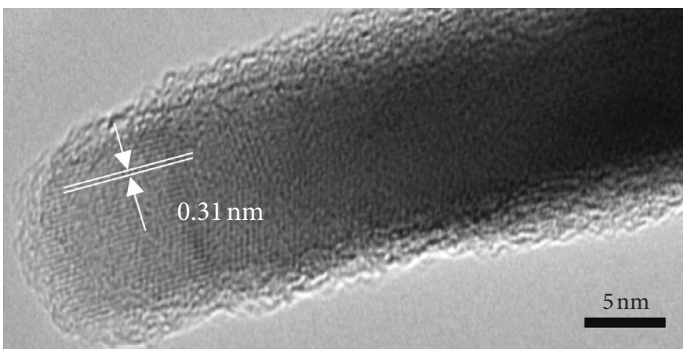

(e)

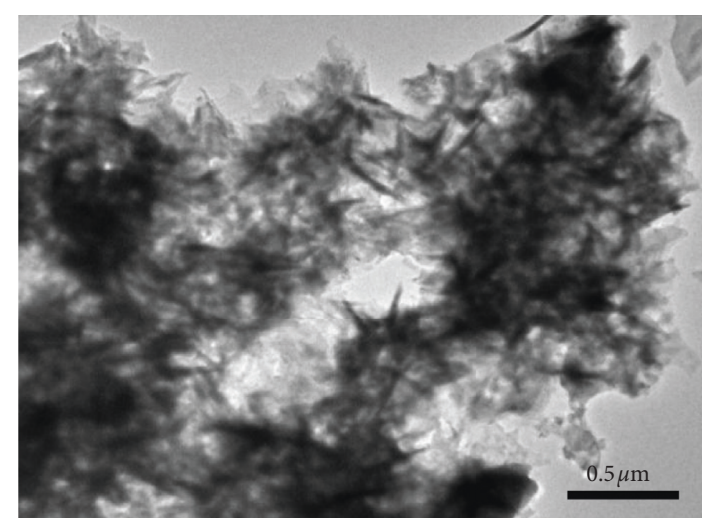

(b)

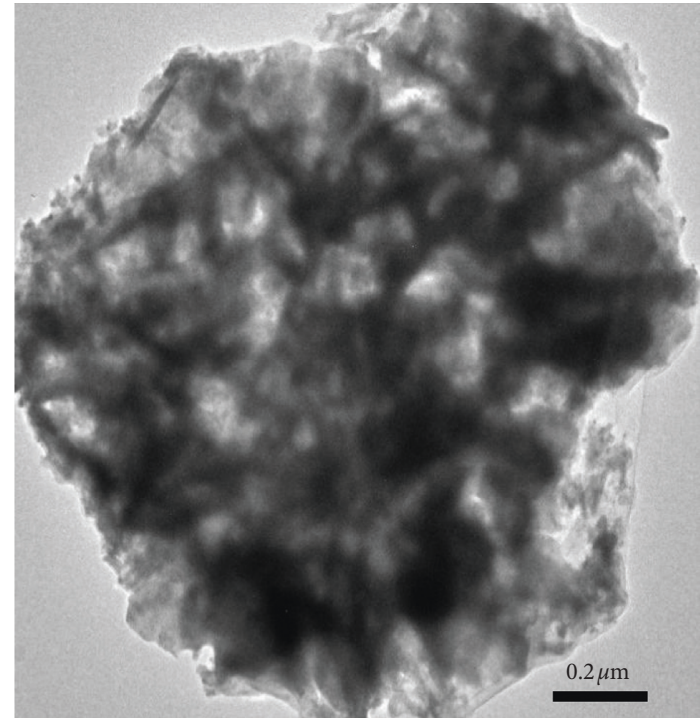

(d)

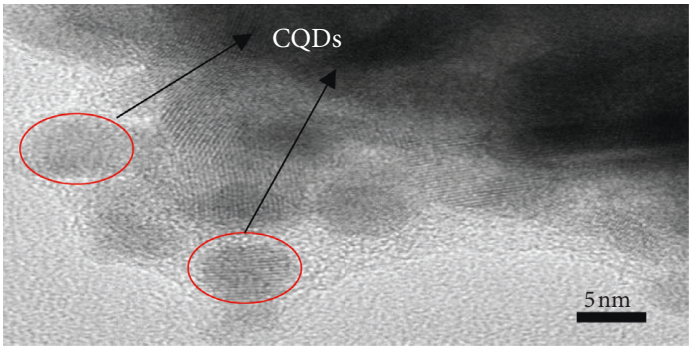

(f)

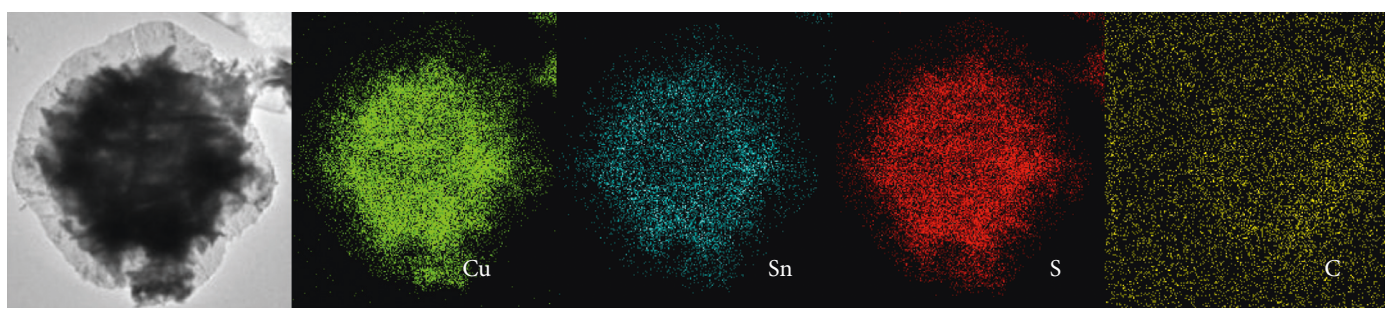

(g)

FIgure 2: Characteristic TEM images of ( $a, c)$ CTSs and (b, d) CTSs@CQDs at low and high magnification. HRTEM images of (e) CTSs and (f) CTSs@CQDs. (g) TEM image of CTSs@CQDs and corresponding elemental mapping results. 


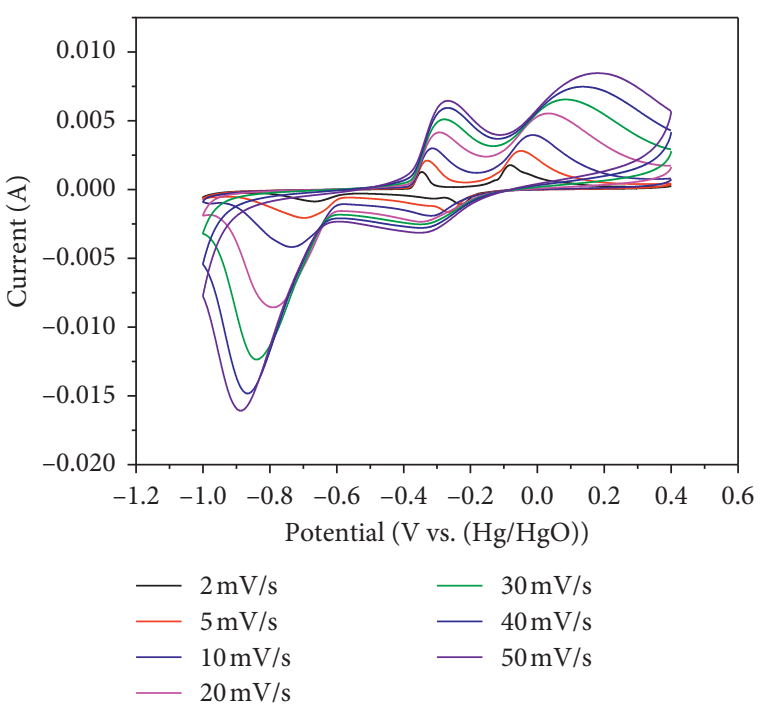

(a)

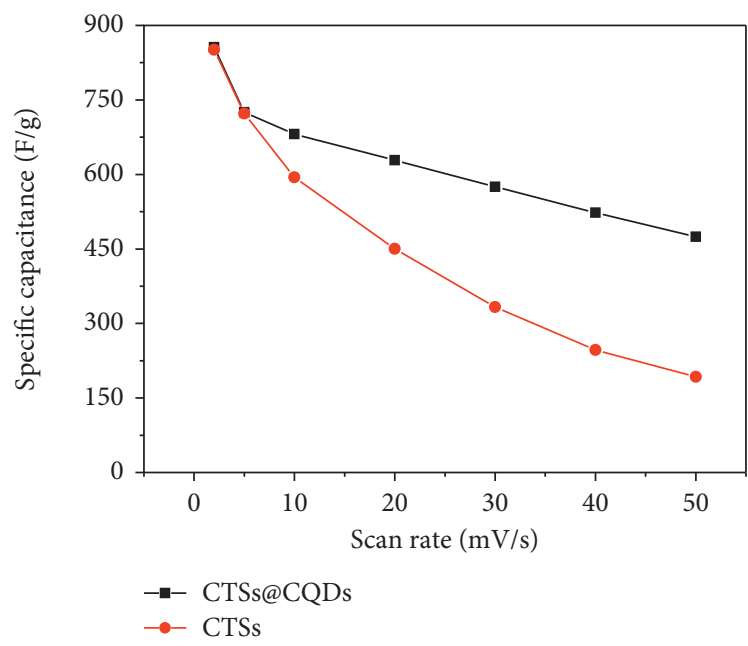

(c)

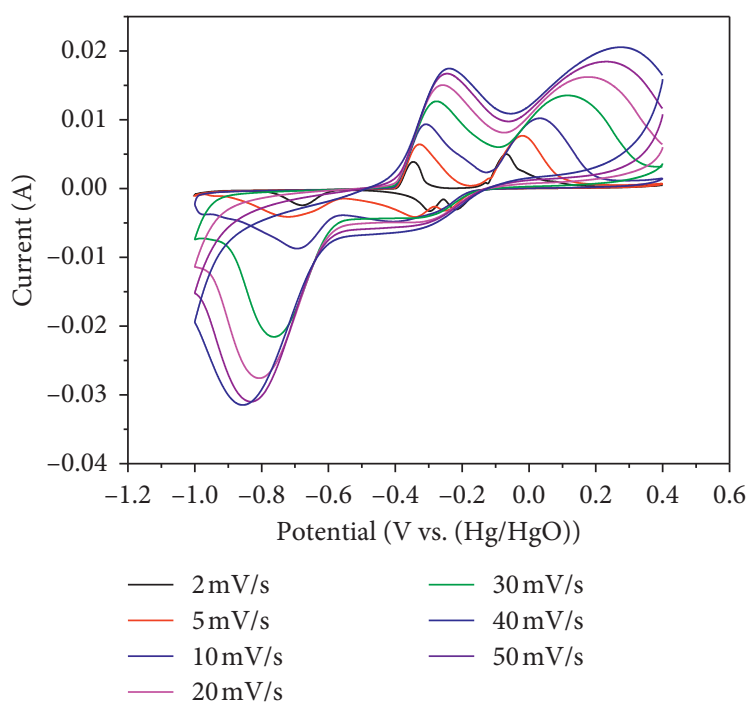

(b)

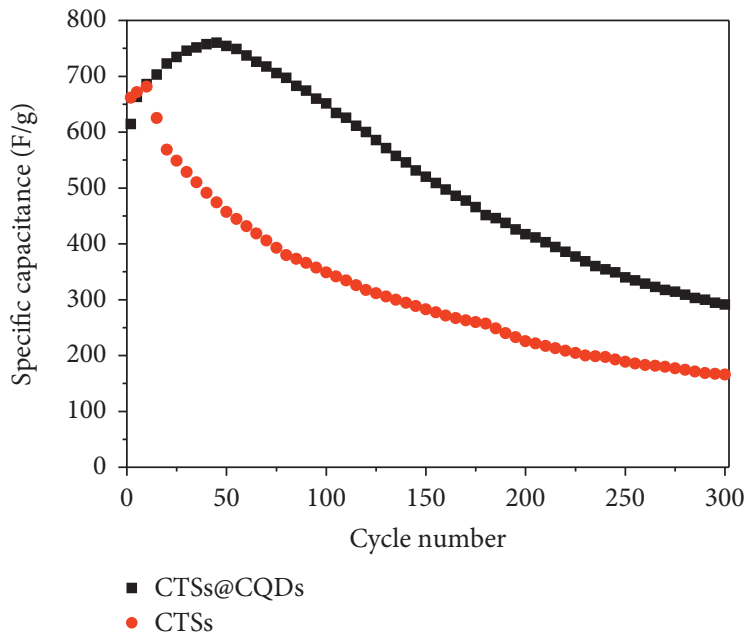

(d)

Figure 3: (a, b) CV curves of CTSs and CTSs@CQDs. (c) Rate capability of CTSs@CQDs and CTSs. (d) Cycling performance of CTSs@ CQDs and CTSs at the current density of $10 \mathrm{~A} \cdot \mathrm{g}^{-1}$.

Figure 3(d). Obviously, CTSs@CQDs displayed superior cycling performance to that of CTSs. It should be noted that the specific capacitance of CTSs@CQDs increased gradually in the initial 50 cycles. This phenomenon might be caused by the activation process of active materials owing to the decoration of CQDs, which not only improved the electrical conductivity of CTSs but also alleviated volume change during redox reactions [28].

\section{Conclusions}

CQDs were employed to modify CTS and yield CTSs@ CQDs with enhanced electrochemical performances. CTSs@CQDs possessed remarkable specific capacitance reaching $856 \mathrm{~F} \cdot \mathrm{g}^{-1}$ at $2 \mathrm{mV} \cdot \mathrm{s}^{-1}$ and a high rate capability of $474 \mathrm{~F} \cdot \mathrm{g}^{-1}$ at $50 \mathrm{mV} \cdot \mathrm{s}^{-1}$. These values were superior to those of CTS $\left(851 \mathrm{~F} \cdot \mathrm{g}^{-1}\right.$ at $2 \mathrm{mV} \cdot \mathrm{s}^{-1}$ and $192 \mathrm{~F} \cdot \mathrm{g}^{-1}$ at $50 \mathrm{mV} \cdot \mathrm{s}^{-1}$, respectively). The improved electrochemical performances of CTSs@CQDs were mainly ascribed to modification of CQDs, which improved the electrical conductivity and alleviated the volume change during redox reactions.

\section{Data Availability}

The data used to support the findings of this study are available from the corresponding author upon request.

\section{Conflicts of Interest}

The authors declare that there are no conflicts of interest regarding the publication of this paper.

\section{Acknowledgments}

The authors acknowledge the financial support from the National Natural Science Foundation of China Program 
(no. 51602111), Natural Science Foundation of Guangdong Province (2018A030313739), Cultivation Project of National Engineering Technology Center (2017B090903008), Xijiang R\&D Team (X.W.), Guangdong Provincial Grant (2017A050506009), Special Fund Project of Science and Technology Application in Guangdong (2017B020240002), and 111 Project.

\section{References}

[1] Y. Li, H. Xie, and J. Tu, "Nanostructured SnS/carbon composite for supercapacitor," Materials Letters, vol. 63, no. 21, pp. 1785-1787, 2009.

[2] C. Shang, S. Dong, S. Wang et al., "Coaxial $\mathrm{Ni}_{x} \mathrm{Co}_{2 x}(\mathrm{OH})_{6 x} /$ TiN nanotube arrays as supercapacitor electrodes," ACS Nano, vol. 7, no. 6, pp. 5430-5436, 2013.

[3] N. Parveen, S. A. Ansari, H. R. Alamri, M. O. Ansari, Z. Khan, and M. H. Cho, "Facile synthesis of $\mathrm{SnS}_{2}$ nanostructures with different morphologies for high-performance supercapacitor applications," ACS Omega, vol. 3, no. 2, pp. 1581-1588, 2018.

[4] Y. Lou, M. Zhang, C. Li et al., "Mercaptopropionic acidcapped wurtzite $\mathrm{Cu}_{9} \mathrm{Sn}_{2} \mathrm{Se}_{9}$ nanocrystals as high-performance anode materials for lithium-ion batteries," ACS Applied Materials \& Interfaces, vol. 10, no. 2, pp. 1810-1818, 2018.

[5] H. Peng, G. Ma, J. Mu, K. Sun, and Z. Lei, "Controllable synthesis of CuS with hierarchical structures via a surfactantfree method for high-performance supercapacitors," Materials Letters, vol. 122, pp. 25-28, 2014.

[6] L. Qian, X. Tian, L. Yang, J. Mao, H. Yuan, and D. Xiao, "High specific capacitance of CuS nanotubes in redox active polysulfide electrolyte," RSC Advances, vol. 3, no. 6, pp. 17031708, 2013.

[7] K.-J. Huang, J.-Z. Zhang, and Y. Fan, “One-step solvothermal synthesis of different morphologies CuS nanosheets compared as supercapacitor electrode materials," Journal of Alloys and Compounds, vol. 625, pp. 158-163, 2015.

[8] S. Ravi, C. V. V. M. Gopi, and H. J. Kim, "Enhanced electrochemical capacitance of polyimidazole coated covellite $\mathrm{CuS}$ dispersed CNT composite materials for application in supercapacitors," Dalton Transactions, vol. 45, no. 31, pp. 12362-12371, 2016.

[9] B. De, J. Balamurugan, N. H. Kim, and J. H. Lee, "Enhanced electrochemical and photocatalytic performance of core-shell CuS@carbon quantum Dots@Carbon hollow nanospheres," ACS Applied Materials \& Interfaces, vol. 9, no. 3, pp. 24592468, 2017.

[10] K. Wang, Y. Huang, Y. Zhu, M. Yu, and M. Wang, "Cubic $\mathrm{Cu}_{2} \mathrm{O} / \mathrm{Cu}_{2} \mathrm{~S}$ particles with a unique truncated edge structure anchoring on reduced graphene oxide as an enhanced anode material for sodium-ion batteries," ChemElectroChem, vol. 5, no. 4, pp. 630-636, 2018.

[11] L. Ma, L. Xu, X. Zhou, X. Xu, and L. Zhang, "Molybdenumdoped few-layered $\mathrm{SnS}_{2}$ architectures with enhanced electrochemical supercapacitive performance," RSC Advances, vol. 5, no. 128, pp. 105862-105868, 2015.

[12] Y. Liu, X.-Y. Yu, Y. Fang et al., "Confining $\mathrm{SnS}_{2}$ ultrathin nanosheets in hollow carbon nanostructures for efficient capacitive sodium storage," Joule, vol. 2, no. 4, pp. 725-735, 2018.

[13] G. Zhang, D. Chen, N. Li et al., " $\mathrm{SnS}_{2} / \mathrm{SnO}_{2}$ heterostructured nanosheet arrays grown on carbon cloth for efficient photocatalytic reduction of Cr(VI)," Journal of Colloid and Interface Science, vol. 514, pp. 306-315, 2018.
[14] H. Chauhan, M. K. Singh, S. A. Hashmi, and S. Deka, "Synthesis of surfactant-free SnS nanorods by a solvothermal route with better electrochemical properties towards supercapacitor applications," RSC Advances, vol. 5, no. 22, pp. 17228-17235, 2015.

[15] W. Wang, H. Cai, G. Chen et al., "Preparation of Sn loss-free $\mathrm{Cu}_{2} \mathrm{SnS}_{3}$ thin films by an oxide route for solar cell," Journal of Alloys and Compounds, vol. 742, pp. 860-867, 2018.

[16] H. D. Shelke, A. C. Lokhande, A. M. Patil, J. H. Kim, and C. D. Lokhande, " $\mathrm{Cu}_{2} \mathrm{SnS}_{3}$ thin film: structural, morphological, optical and photoelectrochemical studies," Surfaces and Interfaces, vol. 9, pp. 238-244, 2017.

[17] L. Fu, X. Wang, J. Ma et al., "Graphene-encapsulated copper tin sulfide submicron spheres as high-capacity binder-free anode for lithium-ion batteries," ChemElectroChem, vol. 4, no. 5, pp. 1124-1129, 2017.

[18] L. Fu, Z. Bi, B. Wei et al., "Flower-like $\mathrm{Cu}_{2} \mathrm{SnS}_{3}$ nanostructure materials with high crystallinity for sodium storage," Nanomaterials (Basel), vol. 8, no. 7, p. 475, 2018.

[19] C. Wang, H. Tian, J. Jiang et al., "Facile synthesis of different morphologies of $\mathrm{Cu}_{2} \mathrm{SnS}_{3}$ for high-performance supercapacitors," ACS Applied Materials \& Interfaces, vol. 9, no. 31, pp. 26038-26044, 2017.

[20] H. Zhang, H. Ming, S. Lian et al., " $\mathrm{Fe}_{2} \mathrm{O}_{3} /$ carbon quantum dots complex photocatalysts and their enhanced photocatalytic activity under visible light," Dalton Transactions, vol. 40, no. 41, pp. 10822-10825, 2011.

[21] J. Di, J. Xia, Y. Ge et al., "Novel visible-light-driven CQDs/Bi 2 $\mathrm{WO}_{6}$ hybrid materials with enhanced photocatalytic activity toward organic pollutants degradation and mechanism insight," Applied Catalysis B: Environmental, vol. 168-169, pp. 51-61, 2015.

[22] X. Jian, J.-G. Li, H.-M. Yang, L.-L. Cao, E.-H. Zhang, and Z.-H. Liang, "Carbon quantum dots reinforced polypyrrole nanowire via electrostatic self-assembly strategy for highperformance supercapacitors," Carbon, vol. 114, pp. 533543, 2017.

[23] X. Chen, R. Paul, and L. Dai, "Carbon-based supercapacitors for efficient energy storage," National Science Review, vol. 4, no. 3, pp. 453-489, 2017.

[24] X. Jian, H.-M. Yang, J.-G. Li, E.-H. Zhang, L.-L. Cao, and Z.-H. Liang, "Flexible all-solid-state high-performance supercapacitor based on electrochemically synthesized carbon quantum dots/polypyrrole composite electrode," Electrochimica Acta, vol. 228, pp. 483-493, 2017.

[25] V. Sharma, S. Biswas, and A. Chandra, "Need for revisiting the use of magnetic oxides as electrode materials in supercapacitors: unequivocal evidence of significant variation in specific capacitance under variable magnetic field," Advanced Energy Materials, vol. 8, no. 22, article 1800573, 2018.

[26] A. Singh, M. A. Akhtar, and A. Chandra, "Trade-off between capacitance and cycling at elevated temperatures in redox additive aqueous electrolyte based high performance asymmetric supercapacitors," Electrochimica Acta, vol. 229, pp. 291-298, 2017.

[27] A. Singh and A. Chandra, "Enhancing specific energy and power in asymmetric supercapacitors-a synergetic strategy based on the use of redox additive electrolytes," Scientific Reports, vol. 6, no. 1, article 25793, 2016.

[28] J. L. Yin and J. Y. Park, "A nickel foam supported copper core/ nickel oxide shell composite for supercapacitor applications," Microporous and Mesoporous Materials, vol. 200, pp. 61-67, 2014. 

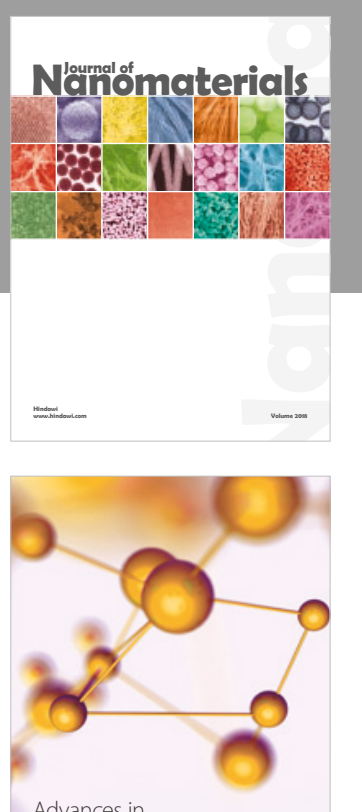

Physical Chemistry
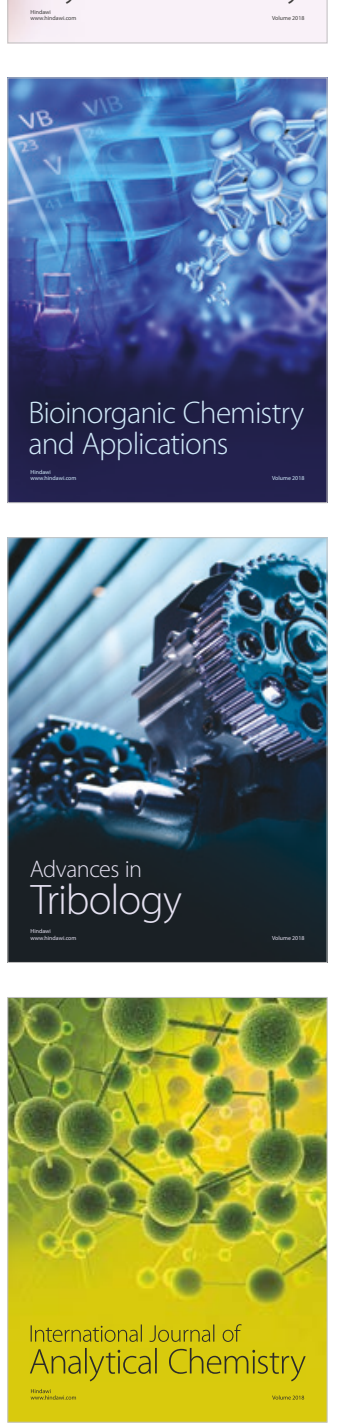

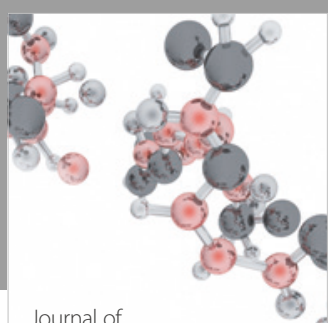

Analytical Methods

in Chemistry

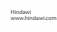

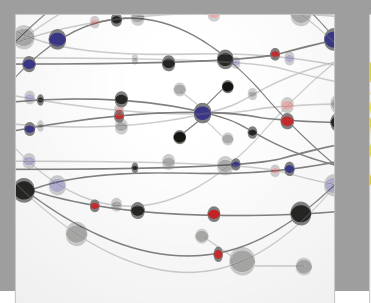

The Scientific World Journal

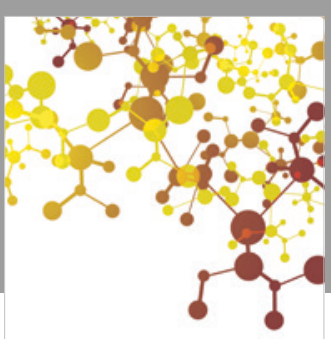

Journal of

Applied Chemistry
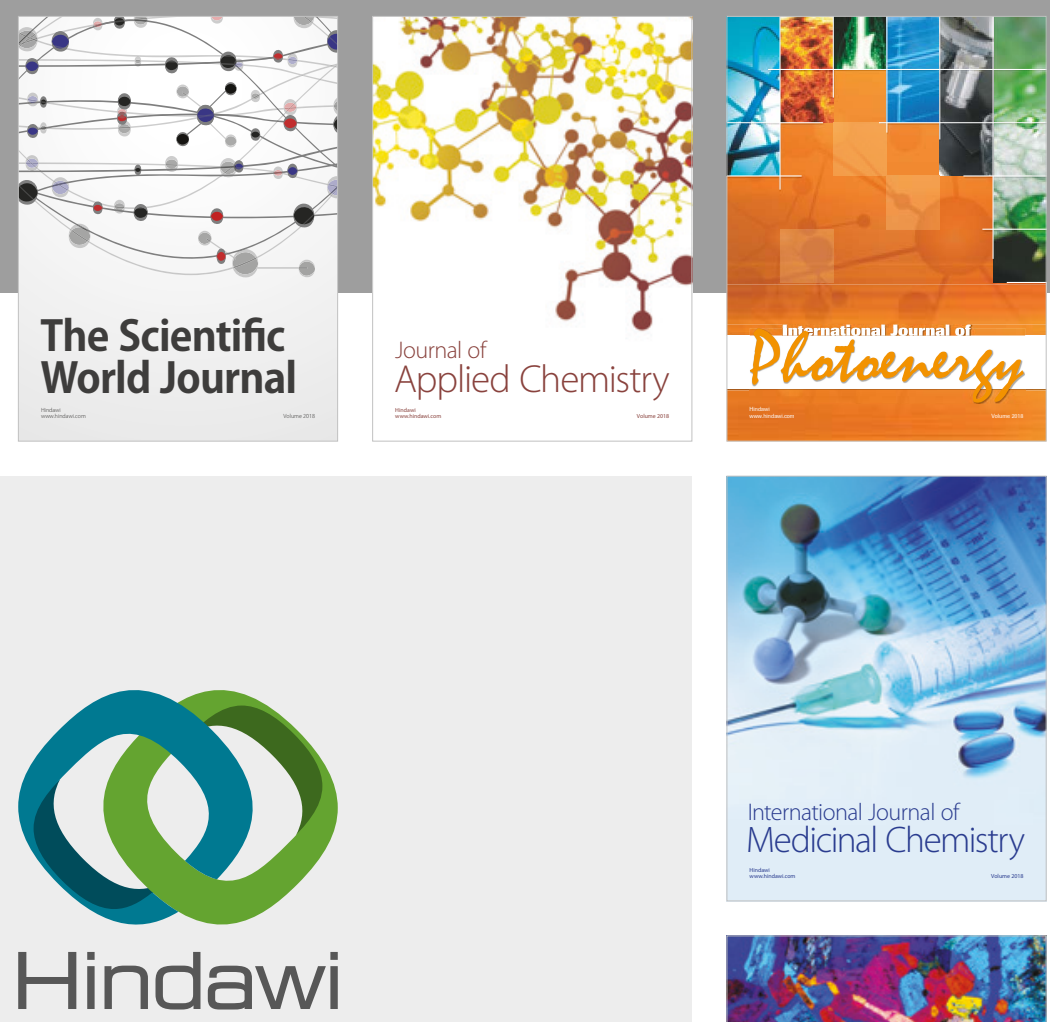

Submit your manuscripts at

www.hindawi.com
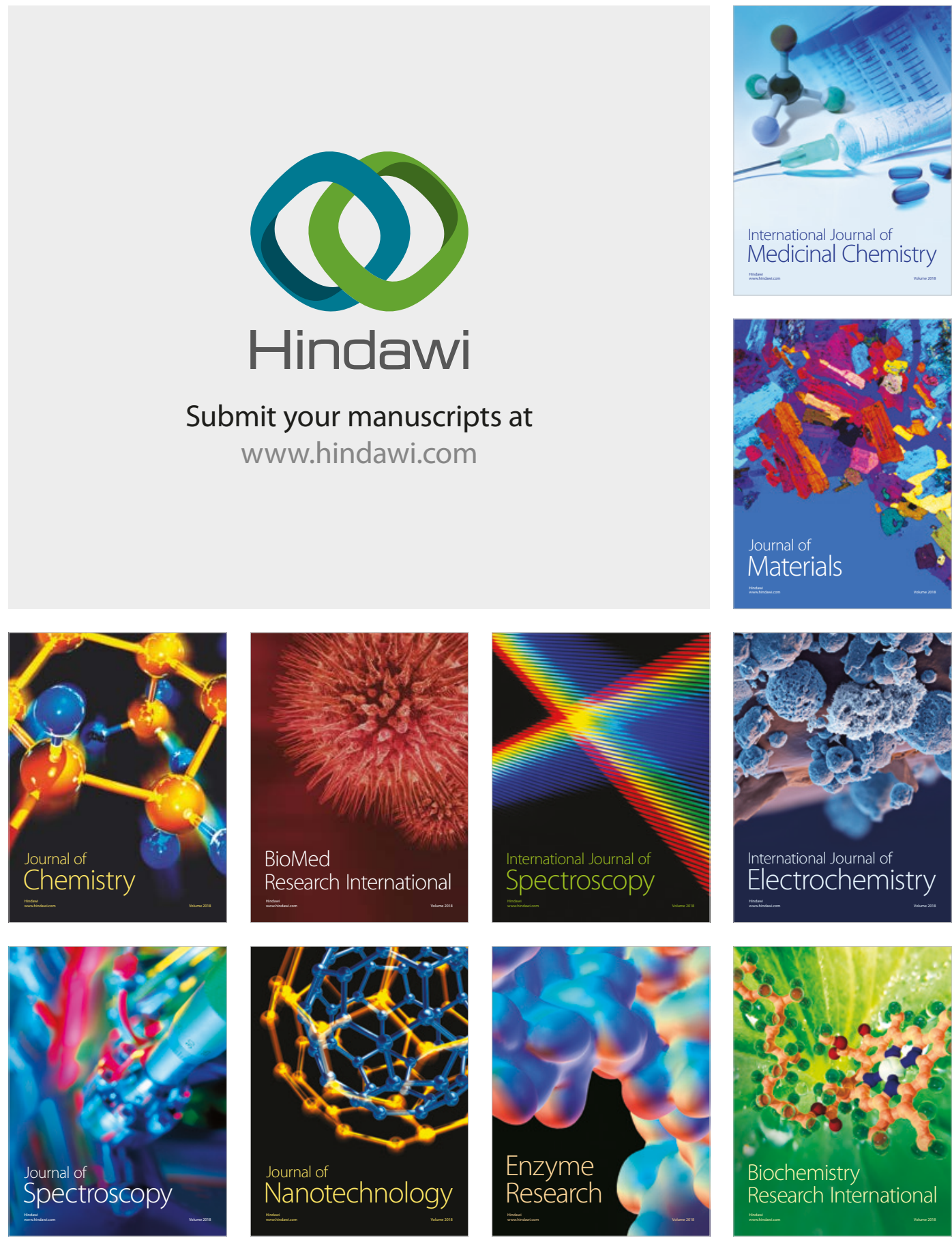
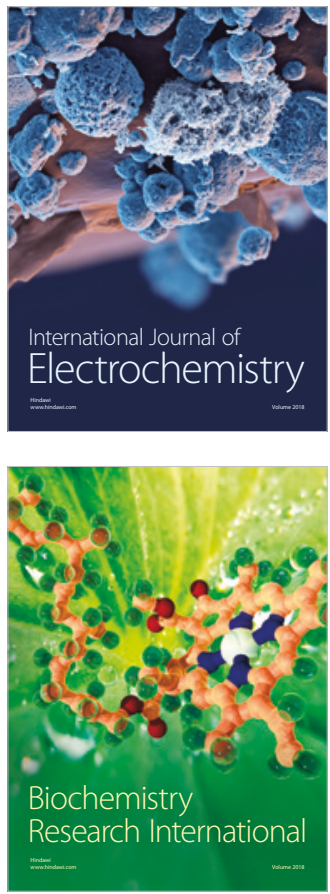